\title{
A case of lipoprotein glomerulopathy with thrombotic microangiopathy due to malignant hypertension
}

\author{
Yu Wu${ }^{1}$, Xiaohan Chen², Yuan Yang ${ }^{3}$, Baohe Wang ${ }^{4}$, Xiaoxia $\mathrm{Liu}^{2}$, Ye Tao ${ }^{2}$, Ping Fu ${ }^{2}$ and Zhangxue $\mathrm{Hu}^{2 *}$
}

\begin{abstract}
Background: Lipoprotein glomerulopathy $(\mathrm{LPG})$ is a rare inherited renal disease characterized by intraglomerular lipoprotein within the lumina of severely dilated glomerular capillaries. The common clinical presentation of LPG includes proteinuria or nephrotic syndrome. Hypertension and anemia were thought to be mild in LPG. Thrombotic microangiopathy (TMA) in LPG has not been previously reported. In this report, we present a patient with LPG that developed TMA. To the best of our knowledge, this is the first report of TMA in LPG.
\end{abstract}

Case presentation: Four years ago (2005), a 19-year-old Chinese woman was diagnosed with nephrotic syndrome and provided prednisone treatment. A combination of prednisone and cyclophosphamide did not have any effect and was discontinued after six months. Although she was steroid-resistant, over the next subsequent three years, she maintained normal renal function without anemia and thrombocytopenia. In February 2009, she had a severe headache and blurry vision and presented at a local hospital with severe hypertension. Blood pressure was 220/ $160 \mathrm{mmHg}$. Laboratory data showed hemoglobin $3.8 \mathrm{~g} / \mathrm{dL}$; platelet counts $29 \times 10^{9} / \mathrm{L}$; urinary protein $7.90 \mathrm{~g} / \mathrm{d}$; total bilirubin $29.9 \mathrm{umol} / \mathrm{L}$; indirect bilirubin $28.2 \mathrm{umol} / \mathrm{L}$; LDH $1172 \mathrm{U} / \mathrm{L} ; A L B 2.66 \mathrm{~g} / \mathrm{dL}$; urea nitrogen $52 \mathrm{mg} / \mathrm{dL}$; serum creatinine $3.2 \mathrm{mg} / \mathrm{dL}$; triglyceride $253 \mathrm{mg} / \mathrm{dL}$; total cholesterol $273 \mathrm{mg} / \mathrm{dL}$. ANA, ds-DNA, ANCA, anti-GBM antibody and anticardiolipin were all negative. A renal biopsy revealed LPG with TMA. Genetic evaluation showed the patient carried the APOE Kyoto mutation. Adequate control of blood pressure improved microangiopathic anemia and thrombocytopenia, however, renal function did not improve and she eventually developed uremia and became hemodialysis dependent.

Conclusion: We report on a rare case of TMA probably due to malignant hypertension in LPG. Early lipid-lowering and antihypertensive treatment may improve outcome. The pathophysiologic relationship between LPG and TMA should be investigated further.

Keywords: Lipoprotein glomerulopathy, Thrombotic microangiopathy, Malignant hypertension, APOE Kyoto

\section{Background}

Lipoprotein glomerulopathy (LPG) is a rare inherited renal disease characterized by the presence of intraglomerular lipoprotein thrombi due to deposition of lipid droplets within the lumina of severely dilated glomerular capillaries, with a variable degree of mesangial proliferation and segmental glomerular sclerosis [1,2]. The common clinical presentation of LPG includes proteinuria or nephrotic

\footnotetext{
* Correspondence: hzxawy@scu.edu.cn

2Department of Nephrology, West China Hospital, National Key Laboratory of Biotherapy of Human Diseases, Sichuan University, Chengdu, Sichuan

Province, China

Full list of author information is available at the end of the article
}

syndrome, which may gradually progress to chronic renal failure. Despite deposition of numerous intraglomerular lipoprotein thrombi, no thrombi in arterioles have been described in LPG patients. Moreover, acute renal failure has rarely developed in LPG patients. In the report herein, we present a case of LPG with thrombotic microangiopathy (TMA). The relationship between LPG and TMA is discussed.

\section{Case presentation}

A 19-year-old Chinese woman was admitted to our hospital in April 2009. Four years prior to this admission, she noticed mild pretibial edema. At that time, urinalysis 
showed proteinuria $3+$. Blood pressure was normal. She was diagnosed with nephrotic syndrome. Oral prednisone was initiated for 2 months with a dosage of $1 \mathrm{mg} / \mathrm{kg}$ per day, then tapered. The total period of prednisone treatment was 6 months. During this period, cyclophosphamide $100 \mathrm{mg}$ per day orally was added for 10 days.
The total cyclophosphamide dosage was $1 \mathrm{~g}$. The patient did not improve with this regimen. During the next 3 years (2005-2008), she maintained stable renal function and normal blood pressure. Six months before admission (November 2008), hypertension developed at 150/90 $\mathrm{mmHg}$. The 24-hour urinary protein was $8.8 \mathrm{~g} / \mathrm{d}$.
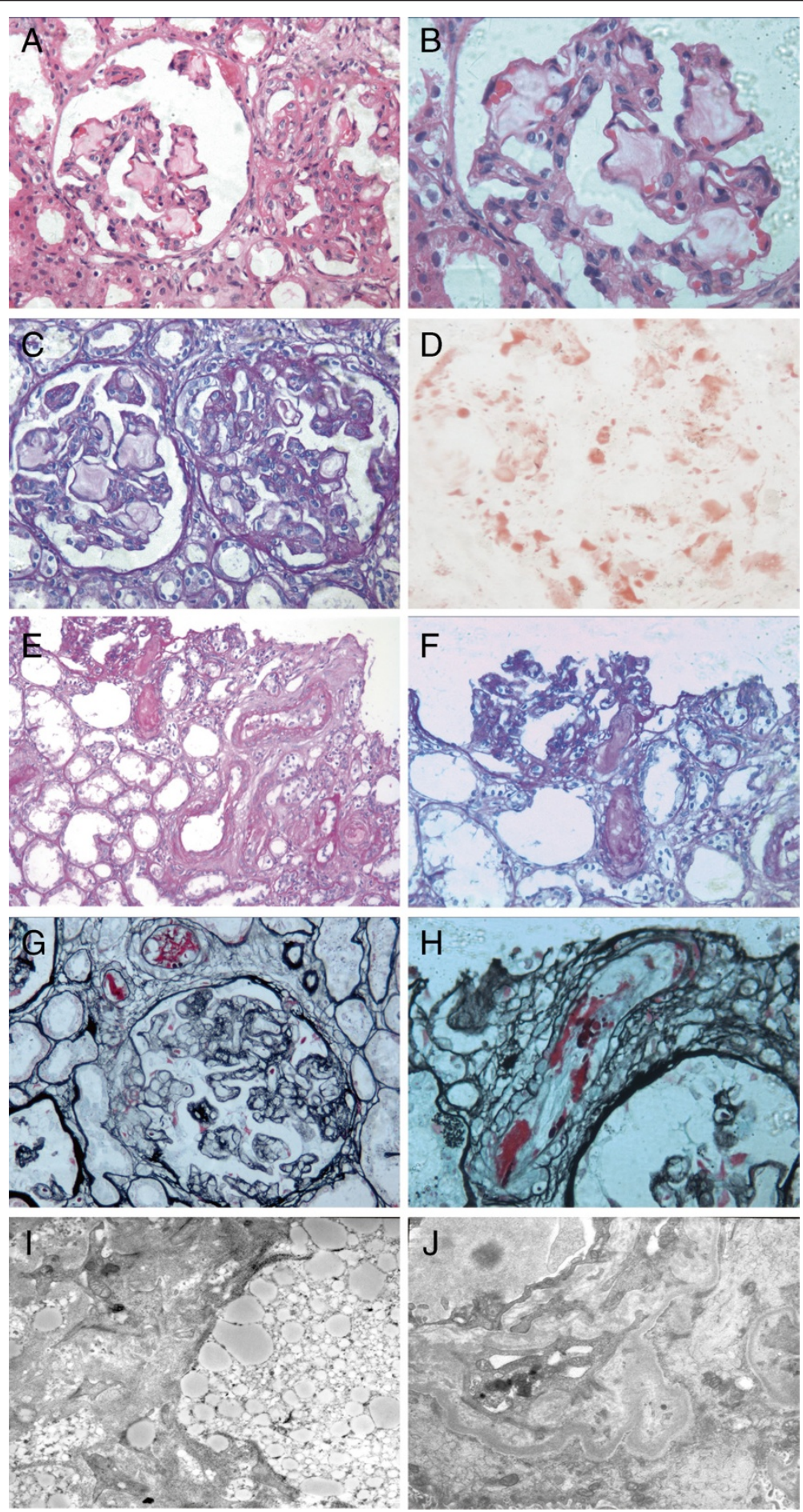

Figure 1 (See legend on next page.) 
(See figure on previous page.)

Figure 1 Glomerular and vascular findings from renal biopsy specimens in a patient with LPG and TMA. Panel A shows a glomerulus with the presence of intraglomerular lipoprotein thrombi due to deposition of lipid droplets within the diluted lumina of glomerular capillaries. Although lipoprotein thrombi were present in glomerular capillaries, the glomerulus still exhibited ischemic features (HE staining; $\times 200)$. In Panel $\mathbf{B}$, the glomerulus shown in Panel $\mathbf{A}$ were enlarged (HE staining; $\times 400)$. In Panel $\mathbf{C}$, glomeruli shown in Panel $\mathbf{A}$ were stained in PAS, also showing intraglomerular lipoprotein thrombi and ischemic features (PAS staining; $\times 200)$. In Panel $\mathbf{D}$, lipid was stained positive (Oil Red $O$ staining; $\times 400)$. In Panel $\mathbf{E}$, a thrombus in the lumina of arteriole which extended to the vascular pole of the adjacent ischemic glomerulus is shown. Other arterioles also showed thrombus and pronounced swelling of endothelial cells (PAS staining; $\times 200$ ). In Panel $\mathbf{F}$, the thrombus in the arteriole extended to the vascular pole of an adjacent ischemic glomerulus shown in Panel $\mathbf{E}$ was enlarged (PAS staining; $\times 400)$. In Panel $\mathbf{G}$, a glomerulus was shown with an extensive double-contour formation. Few red blood cells are observed in the capillaries. Adjacent arterioles with a swelling of endothelial cells and the presence of a fluffy material (fibrin) that caused a narrowing of vascular lumen are shown (PASM staining; $\times 400$ ). In Panel $\mathbf{H}$, endothelial swelling with mucoid change and chunks of fibrin in interlobular arteries are shown. In Panel $\mathbf{I}$, the capillary lumen were occluded with various granule sizes. In Panel $\mathbf{J}$, the subendothelial zone was focally expanded by electron-lucent material, and a new glomerular basement membrane was formed. Panels $\mathbf{I} \& \mathbf{J}$ are electron microscopy images.

Laboratory findings showed a serum total protein of $4.23 \mathrm{~g} / \mathrm{dL}$, serum albumin $2.46 \mathrm{~g} / \mathrm{dL}$, triglyceride $241 \mathrm{mg} /$ $\mathrm{dL}$, total cholesterol $350 \mathrm{mg} / \mathrm{dL}$, HDL-C $71 \mathrm{mg} / \mathrm{dL}$, and LDL-C $233 \mathrm{mg} / \mathrm{dL}$. A complete blood count showed a hemoglobin of $9.8 \mathrm{~g} / \mathrm{dL}, \mathrm{WBC}$ counts $6.6 \times 10^{9} / \mathrm{L}$, and platelet counts $130 \times 10^{9} / \mathrm{L}$. Felodipine $5 \mathrm{mg}$ per day was administered to keep blood pressure at a normal range. Just two months before admission (February 2009), she felt a severe headache, blurry vision, nausea, vomiting, and presented to a local hospital. On admission at that hospital, physical examination showed blood pressure was up to $220 / 160 \mathrm{mmHg}$, with facial pallor, and pitting edema of the lower extremities. Laboratory data showed a hemoglobin of $3.8 \mathrm{~g} / \mathrm{dL}$; WBC counts $3.75 \times 10^{9} / \mathrm{L}$; platelet counts $29 \times 10^{9} / \mathrm{L}$; urinary protein $7.90 \mathrm{~g} / \mathrm{d}$; total bilirubin $29.9 \mathrm{umol} / \mathrm{L}$; indirect bilirubin $28.2 \mathrm{umol} / \mathrm{L}$; ALT 22 U/L; AST 36 U/L; LDH 1172 U/L; ALB 2.66 g/dL; urea nitrogen $52 \mathrm{mg} / \mathrm{dL}$; serum creatinine $3.2 \mathrm{mg} / \mathrm{dL}$; triglyceride $253 \mathrm{mg} / \mathrm{dL}$; total cholesterol $273 \mathrm{mg} / \mathrm{dL}$, HDL-C $46 \mathrm{mg} /$ dL, LDL-C $200 \mathrm{mg} / \mathrm{dL}$, VLDL-C $50 \mathrm{mg} / \mathrm{dL}$, apoA1 $0.90 \mathrm{mg} / \mathrm{dL}$, ароB $1.21 \mathrm{mg} / \mathrm{dL}$. ANA, ds-DNA, ANCA, anti-GBM antibody and anticardiolipin were all negative. HIV, HBV and HCV tests were all negative. Urine HCG was negative. A brain CT scan did not reveal any abnormality. The patient did not have any evidence of malignancy, pregnancy or abortion. During this period, she had not experienced diarrhea or infections. There was no family history of renal diseases, hypertension or hematologic diseases.

A kidney biopsy was performed. Light and electron microscopic examinations of renal biopsy material showed a marked dilatation of the capillary lumen in the glomeruli, and the presence of laminated intraglomerular lipoprotein thrombi due to deposition of lipid droplets within the glomerular capillaries (Figure 1A,B,C). Immunofluorescence showed IgG, IgA, IgM, C3, C4, C1q were all negative. Oil Red O staining was positive (Figure 1D). Electron microscopy showed that the glomerular capillary lumina were occupied by granules of various sizes (Figure 1I). She was diagnosed with LPG and treated with antihypertensive drugs (Nifidipine $60 \mathrm{mg}$ + Prazosin $12 \mathrm{mg}+$ Metoprolol $50 \mathrm{mg}$ per day) and Simvastatin. At the same time, she received hemodialysis and red blood cell transfusion. Over the next four weeks, blood pressure was observed to be under control (average level 135/80 mmHg). Anemia and thrombocytopenia were improved. Hemoglobin was $7.8 \mathrm{~g} / \mathrm{dL}$, platelet counts were $110 \times 10^{9} / \mathrm{L}$; albumin also increased to $3.17 \mathrm{~g} / \mathrm{dL}$; but renal insufficiency was aggravated, with urea nitrogen $24 \mathrm{mg} / \mathrm{dL}$, and serum creatinine $7.2 \mathrm{mg} / \mathrm{dL}$. Urine output decreased progressively to anuria. The patient was then transferred to our hospital.

Because LPG has rarely developed into irreversible acute renal failure, a re-examination of the renal biopsy specimens was performed. Among the 17 glomeruli sampled for light microscopy, three were globally sclerotic, and one was segmental sclerotic. The size of the glomeruli varied greatly. The seven greatly-enlarged glomeruli showed severe dilated capillaries filled with a foamy, whorled, mesh-like material in the glomerular capillary lumens. The other six moderately-enlarged glomeruli showed extensive ischemic changes, retraction of the glomerular tuft and marked thickening and wrinkling of the capillary wall (Figure $1 \mathrm{~A}, \mathrm{~B}, \mathrm{C}$ ). Even in the ischemic glomeruli, a thrombus-like material was observed in some capillaries, so the sizes of these glomeruli were still larger than normal glomeruli, which made the ischemic appearance of these glomeruli hard to identify. Double-contour formations were obvious. Vascular lesions were severe. Swelling of endothelial cells and fluffy masses (fibrin) in arterioles caused narrowing and closure of vascular lumens (Figure 1E,F,G,H). Thrombosis was found in the arterioles, and it extended to the vascular pole of the adjacent ischemic glomerulus, without inflammatory cell infiltration (Figure 1E,F). Under electron microscopy, the glomerular capillaries contained large, intraluminal, finely vacuolated extracellular masses. The vacuoles were predominantly electron lucent, suggesting lipid "thrombi." The subendothelial zone was focally expanded by electron-lucent material (Figure 1I,G). LPG with TMA was diagnosed. In our 
hospital, funduscopic exams revealed significant cotton exudates in both retinas. Serum apoE was $7.2 \mathrm{mg} / \mathrm{dL}$. Serum ADAMTS13 and fecal Shiga toxin were not measured. Although she received fenofibrate $(200 \mathrm{mg}$ per day), hemodialysis, antihypertensive therapy and other supportive therapy, renal failure could not be improved.

Genomic DNA of the patient was extracted from peripheral blood lymphocytes using DNA-isolation kits (TaKaRa, Ostu, Japan). The patient, her sister, and her mother were screened for mutations in the coding sequence of $A P O E$. Four pairs of primers (see Additional file 1: Appendix Table S1) were designed with Primer Premier 5.0 to amplify all exons and adjacent intron/exon boundaries of $A P O E$ (reference sequence GenBank accession no. NM_000041). PCR products containing the sequences of exons 1,2 , and 3 were sequenced directly in both directions, and that of exon 4 was cloned into the pGEM-T vector (Promega, Madison, WI), and sequenced on an ABI377A DNA sequencer (Applied Biosystems, Foster City, CA). APOE Kyoto mutation was identified (Figure 2A). Family study (Figure 2B,C) showed that her mother also carried the $A P O E$ Kyoto mutation, but she did not present any evidence of renal dysfunction, proteinuria or thrombocytopenia. Restriction fragment length polymorphism
(RFLP) analysis for $A P O E$ genotype [3] showed these $A P O E$ Kyoto mutations were located in $\varepsilon 3$ (Figure 2D). Her sister did not carry $A P O E$ Kyoto. Written informed consents were obtained from the patient and her relatives prior to commencement of the genetic analysis.

\section{Conclusions}

Lipoprotein glomerulopathy (LPG) is a rare inherited kidney disease, mainly caused by the $A P O E$ mutation. Less than 80 cases have been reported worldwide since 1989 [4], mainly among persons of Japanese and Chinese origin. Affected patients typically present with nephrotic syndrome, hypertriglyceridemia and elevated plasma apoE levels. Hypertension had been thought to be mild [5]. Approximately half of the reported cases in the literature developed renal failure 1-27 years after onset of disease [6]. Risk factors hastening the progression of LPG have not been clearly identified. On kidney biopsy, intraglomerular lipoprotein thrombi due to deposition of lipid droplets within the lumina of severely dilated glomerular capillaries are the distinct finding for this disease. However, no thrombi in arterioles have been described. TMA has not been previously reported in LPG.

Our case demonstrated LPG and TMA. The patient presented nephrotic syndrome with elevated triglyceride
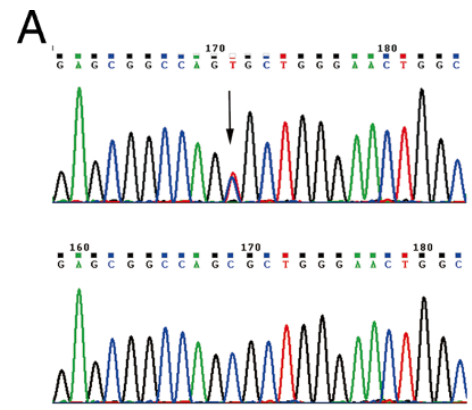

C

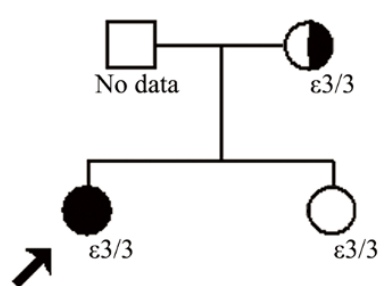

B
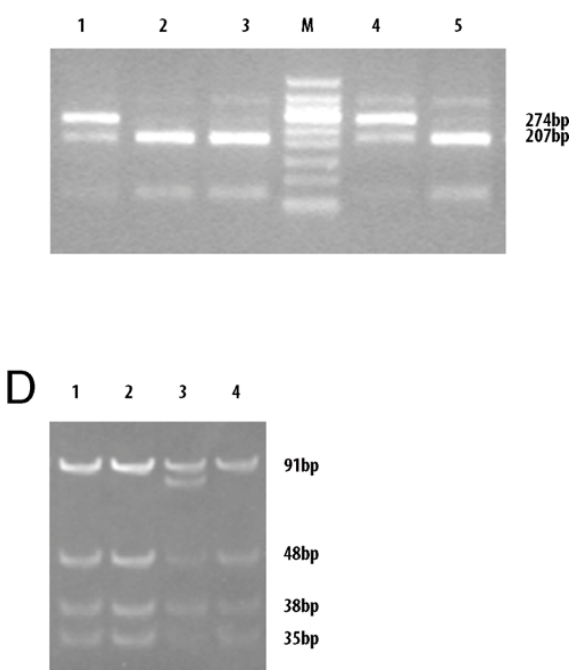

Figure 2 Mutation Detection, Genotyping of ApoE and Family Pedigrees of Patients with Lipoprotein Glomerulopathy (LPG) and TMA. Panel A shows DNA sequencing results of APOE exon 3 of the patient and a healthy control and the positions of the APOE Kyoto mutation (arrow). The LPG patient had a heterozygous C $\rightarrow$ T mutation leading to an amino acid substitution of Cys for Arg at codon 25 of apoE. Panel B shows the results of APOE Kyoto detection with PCR-RFLP analysis. Lanes 1 (the patient) and 4 (patient's mother): the presence of 274 bp and $207 \mathrm{bp}$ fragments indicate heterozygous mutation of APOE Kyoto. Lanes 2 (patient's sister), 3 and 5 (healthy control): the presence of the $207 \mathrm{bp}$ fragment indicates the wild-type homozygote. M: $100 \mathrm{bp}$ molecular ladder. Panel D shows the results of genotyping with PCR-RFLP analysis. Lanes 1 (the patient), 2 (patient's sister), and 4 (patient's mother): the presence of 91 bp, 48 bp, 38 bp and 35 bp fragments indicates an $\varepsilon 3 / 3$ genotype. Lanes 3 (control): the presence of 91 bp, 83 bp, 48 bp, 38 bp and 35 bp fragments indicates an ع2/3 genotype. M: pUC19 DNA/Mspl marker. In Panel C, family pedigree for the patient indicates the APOE Kyoto genotype in probands (arrows) and family members from whom DNA was available. No other family members had clinical evidence of the disease. Men are represented by squares, and women by circles. The shading indicates patients with LPG. The patient's mother is an asymptomatic APOE Kyoto carrier. 
and cholesterol. Light microscopic examinations of renal biopsy material showed a marked dilatation of the capillary lumen in glomeruli with a mesh-like material. Electron microscopy confirmed the presence of extensive lipid thrombi in capillary lumen. LPG had been diagnosed. However, it is hard to attribute acute renal dysfunction, severe anemia and thrombocytopenia to typical LPG. Light and electron microscopic examinations of renal biopsy material showed arteriole thrombosis, endothelial swelling of arterioles, fibrin exudation in the lumina of arterioles, glomerular shrinkage and widening of the subendothelial zone. These thrombi in arterioles were different from lipo-thrombi in glomerular capillaries. The former was stained PAS positive, while the latter had a pale color with PAS and pale green with Masson's trichrome. These thrombi in arterioles should not be the result of a direct deposition of abnormal lipoprotein. Based on elevated blood pressure, acute renal failure, elevated indirect bilirubin and LDH, thrombocytopenia and these renal pathologic findings, LPG with TMA was diagnosed, which has not been previously described in any LPG patient.

TMA is characterized by a syndrome of microangiopathic hemolytic anemia, thrombocytopenia, and variable signs of organ injury due to platelet thrombosis in the microcirculation [7]. TMA usually encompasses hemolytic uremia syndrome (HUS) and thrombotic thrombocytopenic purpura (TTP). TMA can be caused under some predisposing conditions, such as pregnancy, cancer [8], hematopoietic stem cell transplantation [9], HIV infection, systemic lupus erythematosus [10], scleroderma, cyclosporine or antineoplastic treatment [11-13] and malignant hypertension [14,15], which are classified as secondary forms of TMA. It is difficult to distinguish among these diagnoses without clinical information.

Malignant hypertension was defined as severe elevation of mean arterial pressure in combination with grade III to IV hypertensive retinopathy, according to the KeithWagener and Barker classification. It is often accompanied by target organ damage, such as visual disturbances, headache, heart failure, stroke or transient ischemic attack, acute renal dysfunction. TMA was observed in $27 \%$ of patients with malignant hypertension at the emergency department [16]. The pathogenesis of TMA in malignant hypertension is not precisely understood. It is postulated that the endothelial dysfunction caused by activation of the renin-angiotensin-aldosterone system (RAAS) has a central role. Reduction of NO and activation of a coagulation cascade leads to fibrinoid necrosis, edema of arterioles and local platelet aggregation. Microangiopathic hemolysis has been attributed to the mechanical stress when red blood cells pass through the narrowed lumen of arterioles. Rapid control of hypertension could ameliorate TMA and contribute to the improvement of renal function [16].
In this case, the patient suffered severe headache, blurry vision, nausea, vomiting, acute renal failure, grade III hypertensive retinopathy, and microangiopathic hemolytic anemia with blood pressure 220/160 $\mathrm{mmHg}$. Thrombocytopenia, anemia and elevated LDH improved with blood pressure control. All of the above supported the patient suffered TMA which could be triggered by malignant hypertension. Preexisting renal parenchymal diseases were identified in $14 \%$ patients of malignant hypertension [16]. It is reasonable to presume that malignant hypertension was triggered by uncontrolled LPG in this patient.

Most patients with TMA due to malignant hypertension have platelet counts of over $50 \times 10^{9} / \mathrm{L}$ and hemoglobin of over $6.5 \mathrm{~g} / \mathrm{dl}$ [16-18]. In this case, the patient showed severe anemia $(3.8 \mathrm{~g} / \mathrm{dL})$ and thrombocytopenia $(29 \times$ $10^{9} / \mathrm{L}$ ), which is very rare in TMA due to malignant hypertension. As is known, there is a considerable amount of lipoprotein thrombi in intraglomerular capillaries in LPG, and blood passes through the narrow space between the capillary wall and thrombus (Figure 1). We presume that in malignant hypertension, the above mentioned lipoprotein thrombi led red blood cells to be more vulnerable to shear stress, which could aggravate microangiopathic hemolysis and worsen renal function. However, in view of severe thrombocytopenia and anemia in this patient and limited LPG cases reported, the connection between LPG and TMA may be coincidental and need further investigation.

Malignant hypertension with TMA is a medical emergency. Its outcome depends on adequate control of blood pressure and serum creatinine on presentation [19]. For this case, the control of hypertension ameliorated microangiopathic hemolytic anemia, but did not improve renal failure. For LPG patients, early renal biopsy is crucial, because an early accurate diagnosis and treatment of LPG shows remission and survival benefit. Protein A immunoadsorption [20] and LDL-apheresis [21] could induce remission of LPG, but are very expensive. Treatment with intensive lipid-lowering agents, including fibrates, has been reported to lead to clinical remission along with histological resolution of lipoprotein thrombi in serial biopsies [22-25]. Clinical remission of LPG may reduce the risks of malignant hypertension with TMA. Therefore, early biopsy, effective treatment (fibrate-containing treatment), and adequate control of blood pressure are important to improving the outcome of LPG.

Previous literature has not reported that cyclophosphamide alone with moderate dosage can induce TMA $[26,27]$. This patient only received cyclophosphamide with a total dosage of $1 \mathrm{~g}$ and had maintained stable renal function, normal hemoglobin and platelet count over the next three years. Therefore, for this patient, cyclophosphamide did not appear to be the cause of TMA three years later. 
In summary, we report herein a rare case of LPG that developed severe TMA due to malignant hypertension. Adequate control of blood pressure ameliorated microangiopathic hemolytic anemia, but not renal failure. For LPG, early diagnosis and treatment may lower the risk of malignant hypertension with TMA. The factors that may trigger this course and how patients should be treated has not yet been determined. Careful observations of renal tissue under light and electronic microscopy, especially for arterioles are key to diagnosing LPG with TMA.

\section{Consent}

Written informed consents were obtained from the patient and her relatives for publication of this case report and any accompanying images.

\section{Additional file}

Additional file 1: Appendix Table S1. Primer Sequences, Annealing Temperatures, and Product Sizes of the PCR Products.

\section{Abbreviations}

LPG: Lipoprotein glomerulopathy; TTP: Thrombotic thrombocytopenic purpura; HUS: Hemolytic uremic syndrome; TMA: Thrombotic microangiopathy.

\section{Competing interests}

The authors declare that they have no competing interests.

\section{Authors' contributions}

YW, XHC, BHW, XXL, YT, PF, ZXH were the physicians treating the patient in this report. BHW and $Z X H$ performed the renal biopsy evaluation and the pathology studies. $Y Y$ performed genetic studies. The manuscript was prepared by YW, XHC, BHW, YY, YT, PF and ZXH. All authors participated in discussions about the manuscript and approved the final version.

\section{Acknowledgements}

This work was supported by grants from the National Key Technologies R\&D program (Grant number: 2006BAI05A08, PR. China), the National Natural Science Foundation of China (81100330/H0801) and Sichuan Province Key Technologies R\&D program (Grant number: 2009SZ0143, 2010FZ0046).

\section{Author details}

'Department of Hematology, West China Hospital, National Key Laboratory of Biotherapy of Human Diseases, Sichuan University, Chengdu, Sichuan Province, China. ${ }^{2}$ Department of Nephrology, West China Hospital, National Key Laboratory of Biotherapy of Human Diseases, Sichuan University, Chengdu, Sichuan Province, China. ${ }^{3}$ Department of Medical Genetics, West China Hospital, National Key Laboratory of Biotherapy of Human Diseases, Sichuan University, Chengdu, Sichuan Province, China. ${ }^{4}$ Department of Nephrology, Nuclear industry 416 Hospital, Chengdu, Sichuan Province, China.

Received: 8 September 2012 Accepted: 13 February 2013 Published: 28 February 2013

\section{References}

1. Liberopoulos E, Siamopoulos K, Elisaf M: Apolipoprotein E and renal disease. Am J Kidney Dis 2004, 43(2):223-233.

2. Saito T, Ishigaki Y, Oikawa S, Yamamoto TT: Etiological significance of apolipoprotein E mutations in lipoprotein glomerulopathy. Trends Cardiovasc Med 2002, 12(2):67-70.

3. Hixson JE, Vernier DT: Restriction isotyping of human apolipoprotein $\mathrm{E}$ by gene amplification and cleavage with Hhal. J Lipid Res 1990, 31(3):545-548.
4. Saito T, Sato H, Kudo K, Oikawa S, Shibata T, Hara Y, Yoshinaga K, Sakaguchi $\mathrm{H}$ : Lipoprotein glomerulopathy: glomerular lipoprotein thrombi in a patient with hyperlipoproteinemia. Am J Kidney Dis 1989, 13(2):148-153.

5. Saito T, Matsunaga A, Oikawa S: Impact of lipoprotein glomerulopathy on the relationship between lipids and renal diseases. Am J Kidney Dis 2006, 47(2):199-211

6. Sam R, Wu H, Yue L, Mazzone T, Schwartz MM, Arruda JA, Dunea G, Singh AK: Lipoprotein glomerulopathy: a new apolipoprotein $\mathrm{E}$ mutation with enhanced glomerular binding. Am J Kidney Dis 2006, 47(3):539-548.

7. Ruggenenti P, Cravedi P, Remuzzi G: Thrombotic microangiopathies, including hemolytic uremic syndrome. In Comprehensive clinical nephrology. 4th edition. Edited by Floege J, Johnson RJ, Feehally J. Missouri: Elsevier Saunders; 2010:344-355.

8. Yokomine T, Hirakawa H, Ozawa E, Shibata K, Nakayama T: Pulmonary thrombotic microangiopathy caused by gastric carcinoma. J Clin Pathol 2010, 63(4):367-369.

9. Mii A, Shimizu A, Masuda Y, Fujino T, Kaneko T, Utsumi K, Arai T, Ishikawa A, Wakamatsu K, Tajika K, et al: Renal thrombotic microangiopathy associated with chronic humoral graft versus host disease after hematopoietic stem cell transplantation. Path Int 2011, 61(1):34-41.

10. Hu WX, Liu ZZ, Chen HP, Zhang HT, Li LS, Liu ZH: Clinical characteristics and prognosis of diffuse proliferative lupus nephritis with thrombotic microangiopathy. Lupus 2010, 19(14):1591-1598.

11. Dlott JS, Danielson CFM, Blue-Hnidy DE, MCCarthy LJ: Drug-induced thrombotic thrombocytopenic purpura/hemolytic uremic syndrome: a concise review. Ther Apher Dial 2004, 8(2):102-111.

12. George JN, Raskob GE, Shah SR, Rizvi M, Hamilton S, Osborne S, Vondracek T: Drug-induced thrombocytopenia: a systematic review of published case reports. Ann Intern Med 1998, 129(11):886-890.

13. Jackson AM, Rose BD, Graff LG, Jacobs JB, Schwartz JH, Strauss GM, Yang JP, Rudnick MR, Elfenbein IB, Narins RG: Thrombotic microangiopathy and renal failure associated with antineoplastic chemotherapy. Ann Intern Med 1984, 101(1):41-44

14. Akimoto T, Muto S, Ito C, Takahashi H, Takeda S, Ando Y, Kusano E: Clinical features of malignant hypertension with thrombotic microangiopathy. Clin Exp Hypertens 2011, 33(2):77-83.

15. Pardo V, Aburano A: Thrombotic microangiopathy and malignant hypertension. Am J Clin Pathol 1966, 45(5):605-613.

16. van den Born BJ, Honnebier UP, Koopmans RP, van Montfrans GA: Microangiopathic hemolysis and renal failure in malignant hypertension. Hypertension 2005, 45(2):246-251.

17. van den Born BJ, van der Hoeven NV, Groot E, Lenting PJ, Meijers JC, Levi $M$, van Montfrans GA: Association between thrombotic microangiopathy and reduced ADAMTS13 activity in malignant hypertension. Hypertension 2008, 51(4):862-866

18. Shibagaki Y, Fujita T: Thrombotic microangiopathy in malignant hypertension and hemolytic uremic syndrome (HUS)/ thrombotic thrombocytopenic purpura (TTP): can we differentiate one from the other? Hypertens Res 2005, 28(1):89-95.

19. Lip GY, Beevers M, Beevers DG: Complications and survival of 315 patients with malignant-phase hypertension. J Hypertens 1995, 13(8):915-924.

20. Xin Z, Zhihong L, Shijun L, Jinfeng Z, Huiping C, Caihong Z, Daxi J, Leishi L: Successful treatment of patients with lipoprotein glomerulopathy by protein A immunoadsorption: a pilot study. Nephrol Dial Transplant 2009, 24(3):864-869.

21. Russi G, Furci $L$, Leonelli M, Magistroni R, Romano N, Rivasi P, Albertazzi A: Lipoprotein glomerulopathy treated with LDL-apheresis (heparininduced extracorporeal lipoprotein precipitation system): a case report. J Med Case Reports 2009, 3:9311.

22. Hamatani H, Hiromura K, Kobatake K, Yoshida H, Kobayashi S, Yoneda N, Kayakabe K, Matsumoto T, Kuroiwa T, Ueki K, et al: Successful treatment of lipoprotein glomerulopathy in a daughter and a mother using niceritrol. Clin Exp Nephrol 2010, 14(6):619-624.

23. Matsunaga A, Furuyama M, Hashimoto T, Toyoda K, Ogino D, Hayasaka K: Improvement of nephrotic syndrome by intensive lipid-lowering therapy in a patient with lipoprotein glomerulopathy. Clin Exp Nephrol 2009, 13(6):659-662.

24. leiri N, Hotta O, Taguma Y: Resolution of typical lipoprotein glomerulopathy by intensive lipid-lowering therapy. Am J Kidney Dis 2003, 41(1):244-249.

25. Arai T, Yamashita S, Yamane M, Manabe N, Matsuzaki T, Kiriyama K, Kanayama Y, Himeno S, Matsuzawa Y: Disappearance of intraglomerular lipoprotein thrombi and marked improvement of nephrotic syndrome 
by bezafibrate treatment in a patient with lipoprotein glomerulopathy. Atherosclerosis 2003, 169(2):293-299.

26. Fisher DC, Sherrill GB, Hussein A, Rubin P, Vredenburgh JJ, Elkordy M, Ross M, Petros W, Peters WP: Thrombotic microangiopathy as a complication of high-dose chemotherapy for breast cancer. Bone Marrow Transplant 1996, 18(1):193-198

27. Porta C, Danova M, Riccardi A, Bobbio-Pallavicini E, Ascari E: Cancer chemotherapy-related thrombotic thrombocytopenic purpura: biological evidence of increased nitric oxide production. Mayo Clin Proc 1999, 74(6): 570-574.

doi:10.1186/1471-2369-14-53

Cite this article as: Wu et al.: A case of lipoprotein glomerulopathy with thrombotic microangiopathy due to malignant hypertension. BMC Nephrology 2013 14:53.

\section{Submit your next manuscript to BioMed Central and take full advantage of:}

- Convenient online submission

- Thorough peer review

- No space constraints or color figure charges

- Immediate publication on acceptance

- Inclusion in PubMed, CAS, Scopus and Google Scholar

- Research which is freely available for redistribution 